\title{
How bees distinguish black from white
}

This article was published in the following Dove Press journal:

Eye and Brain

31 October 2014

Number of times this article has been viewed

\section{Adrian Horridge}

Biological Sciences, Australian National University, Canberra, ACT, Australia
Correspondence: Adrian Horridge

76 Mueller Street, Yarralumla,

ACT 2600, Australia

Email horridge@netspeed.com.au
Abstract: Bee eyes have photoreceptors for ultraviolet, green, and blue wavelengths that are excited by reflected white but not by black. With ultraviolet reflections excluded by the apparatus, bees can learn to distinguish between black, gray, and white, but theories of color vision are clearly of no help in explaining how they succeed. Human vision sidesteps the issue by constructing black and white in the brain. Bees have quite different and accessible mechanisms. As revealed by extensive tests of trained bees, bees learned two strong signals displayed on either target. The first input was the position and a measure of the green receptor modulation at the vertical edges of a black area, which included a measure of the angular width between the edges of black. They also learned the average position and total amount of blue reflected from white areas. These two inputs were sufficient to help decide which of two targets held the reward of sugar solution, but the bees cared nothing for the black or white as colors, or the direction of contrast at black/white edges. These findings provide a small step toward understanding, modeling, and implementing in silicon the anti-intuitive visual system of the honeybee, in feeding behavior.

Keywords: vision, detectors, black/white, color, visual processing

\section{Introduction}

There has been much interest in how bees detect the shapes and colors of flowers and of landmarks by which they recognize places, but the way that they distinguish between black and white appears to have been overlooked. The three types of photoreceptors of the bee compound eye have spectral sensitivity peaks in the green, blue, and ultraviolet (UV) wavelengths, ${ }^{1}$ so it has usually been accepted that color is detected from the interaction of the responses in these three channels. ${ }^{2,3}$ However, the strength of the stimulus at each receptor type tells nothing about the subsequent neural processing. All three receptor types respond to white and shades of gray, including UV, and the bee's preferences for colors are indifferent to sunlight or shade. However, the rapid changes of sensitivity over a 1,000-fold range seen with changes of light intensity, ${ }^{4}$ make it unlikely that the bee's discrimination of gray, white, or color is based simply on the ratios of the intensities at the receptors.

A good deal is already known about the peripheral neural input pathways. Electrophysiology shows that the responses to steady illumination adapt rapidly. ${ }^{5}$ The responses of the photoreceptors, and particularly of the second-order neurons, are rapidly changing potentials (phasic responses), called modulations. ${ }^{5}$ Bees detect changes of intensity caused by scanning across edges. In brief, the spatial and temporal properties 
of the peripheral neurons are optimized to detect moving stimuli and edges, ${ }^{6}$ not areas of black, white, or color.

In return for a reward of sugar solution, bees readily learn to come to the "choice" chamber where they can choose between the patterns or colors displayed on two targets (Figure 1). Earlier tests of trained bees in the apparatus showed that the positions of the black or gray panels were located very well in the vertical direction, but poorly along the horizontal, ${ }^{7}$ despite the abundant contrast at the vertical edges. Even more curious, a black/white edge was not distinguished from a white/black edge, ${ }^{8}$ which suggests that the edge detectors are symmetrical.

In previous work with black/white patterns, bees discriminated mainly by the modulation of the green receptor pathway. They detected edge orientation and radial and circular edges as components of patterns. ${ }^{9}$ They showed no evidence that patterns or shapes were reassembled, and plenty to show that they did not. The bees measured modulations of the blue and green receptors separately ${ }^{10}$ and recognized them only in the positions where they had been learned on the retina. ${ }^{11}$

Therefore, with UV excluded from the experiments, there are four independent inputs at each local region of the eye that could respond to a white, gray, or colored pattern. These are the green receptor intensity; the blue receptor intensity, called tonic responses; and also the respective changes (modulation) of intensity at each of these receptors, called

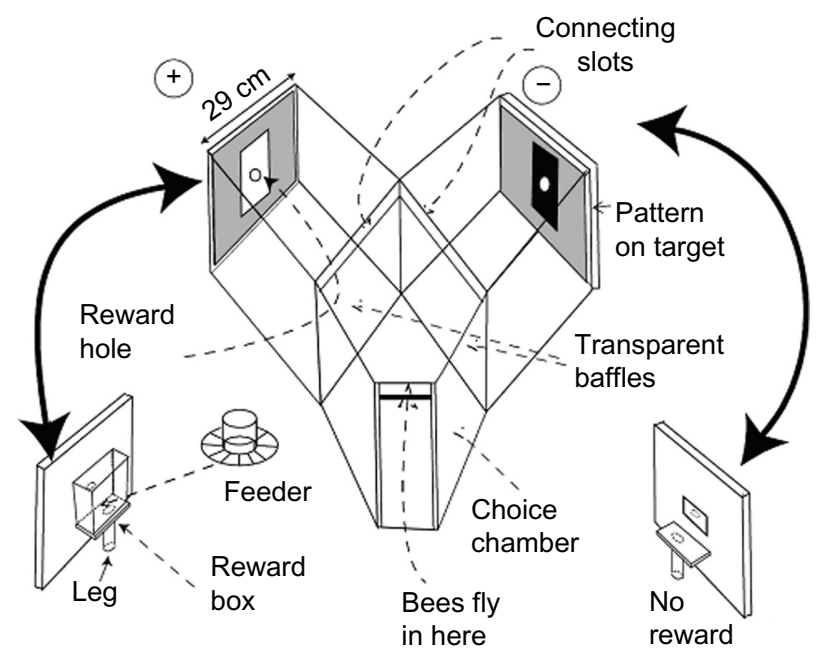

Figure I This Y-choice apparatus was used in all the experiments. The bees fly in at the front and make a choice at a fixed distance from the two targets. The criterion for success is when the bee passes over one of the transparent baffles. The two targets, together with the reward, change sides every 5 minutes. The bees exit by the way they entered.

Note: Insets - the targets seen from behind, showing the support legs and the reward box behind one of them. phasic responses. The aim of this study was to discover which of these inputs the bees use for black versus white on gray backgrounds.

\section{Materials and methods}

The way to train honeybees and to test their preferences in forced choices between carefully selected pairs of test patterns has been described many times. ${ }^{4,7-11}$ The bees used in the study were ordinary brown honeybees (Apis mellifera L.) flying freely from a neighboring hive for 9 months of the year. Ten to fifteen bees were individually color-marked, and only these were allowed to enter the apparatus (Figure 1). A greater number of bees would have risked visual contact between bees, and this matters (only) in the tests.

The apparatus (Figure 1) was made with wooden sides and a transparent polycarbonate sheet top which excluded UV light. Further protection was provided by a transparent plastic roof over the whole. The floor and inside walls of the apparatus were painted white. The reward was a solution of cane sugar that was adjusted in strength between $2 \%$ and $7 \% \mathrm{w} / \mathrm{w}$ so that marked bees continued to return for more, but recruits were not attracted to the weaker solution. The test pattern and the reward changed sides every 5 minutes (Figure 1) to prevent bees from learning which side to go to and to equalize any chance of spurious cues from unequal olfactory cues or side preferences. The reward was provided during the test phase, so that the bees would not continue to search. Test phases were for 5 minutes, with intervening continued training for 20 minutes. At a different time, the test was repeated with the two sides reversed, and each test was followed by a different test pattern. No one test was repeated in the same day. Training and testing continued all week until sufficient counts (100 or 200) accumulated.

Canson (Annonay, France) colored papers were used during tests. These can be ordered online (www.canson-infinity.

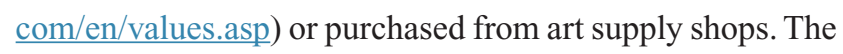
manufacturer names of the colored papers used, and details of the spectra's calibration of sunlight reflected from these papers are given in Table 1. The methods of calibrating the papers are available in the literature. ${ }^{7,12,13}$

Statistics are scarcely necessary because the training score depends on the length of training, the tests were designed so that the results were unambiguous, and a variety of different training patterns with numerous tests supported each other. The decisions of the bees are independent, and the scores are 
Table I Relative receptor stimuli from the different papers, relative to the white paper (100\%), and contrasts between two pairs of papers

\begin{tabular}{lll}
\hline Canson colored papers & Blue receptor (\%) & $\begin{array}{l}\text { Green } \\
\text { receptor (\%) }\end{array}$ \\
\hline Hemp 374 & 34.2 & 56.3 \\
Ultramarine 590 & 33.8 & 20.7 \\
Billiards green 576 & 17.0 & 22.3 \\
Buff 384 & 25.7 & 41.7 \\
Blue 595 & 54.2 & 40.0 \\
White copy paper & 100 & 100 \\
Contrast 374/590 & 0.006 & 0.46 \\
Contrast 384/595 & 0.36 & 0.02 \\
\hline
\end{tabular}

Note: Color names and numbers are those used by the manufacturer, Canson (Annonay, France; www.canson-infinity.com/en/values.asp).

frequencies, so the standard deviation (SD) can be calculated from the formula

$$
\mathrm{SD}=\sqrt{ }\{(\mathrm{p}) \cdot(1-\mathrm{p}) / \mathrm{n}\}
$$

where $\mathrm{p}$ is the fraction of correct choices and $\mathrm{n}$ is the number of choices counted. ${ }^{8}$ A score of $60 \%$ correct with $n=100$, or $57 \%$ correct with $n=200$ was considered significant at the $5 \%$ level. In some of the interesting critical tests where the bees failed, the score was near $50 \%$, but that can be a meaningful result, leading to new conclusions. Care and common sense is required when comparing scores because each test is a forced choice between two unfamiliar targets. A poor score may mean poor learning in the training or little to distinguish in the test. The most informative tests were when the bees failed and the missing input could be supplied in a further test.

\section{Results}

\section{White on gray is easily distinguished from black on gray}

The first experimental training patterns (Figure 2A) were rectangles subtending $16^{\circ} \times 35^{\circ}$ on a background of gray $(50 \%$ black). The stimulus difference between white on gray and black on gray was the same but in opposite directions, and the rewarded target reflected more light. The bees did not learn the contrast at the outer edges because it was the same on both targets. Learning was rapid to a high score. The trained bees failed to distinguish the rewarded training target from a plain gray target (Figure 2B), showing that they did not recognize the white panel nor prefer the lighter target. On the other hand, the plain gray target was distinguished very well from the black panel on gray (score $81 \%$ correct) (not illustrated), showing that the strongest preferred memory originated from

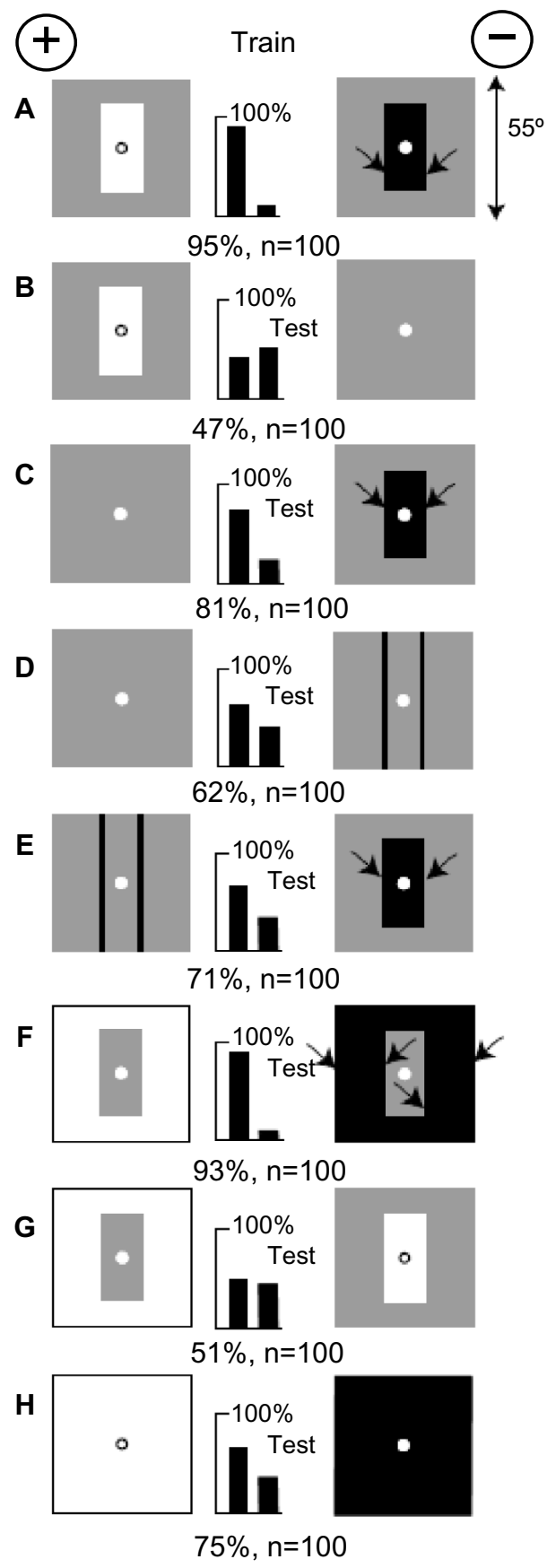

Figure 2 White was distinguished from black on a gray background.

Notes: (A) Training patterns. (B) The trained bees failed to distinguish the white panel from plain gray. (C) They had learned the black panel very well. (D and E) They avoided the vertical parallel lines but the black panel even more so. (F) Reversing the contrast of the panels made little difference to the score. (G) They failed to distinguish a gray panel on white from the rewarded white panel on gray. $(\mathbf{H})$ The trained bees avoided the contrast at black edges (arrows).

the black panel. Learning the unrewarded target first is a consequence of the learning by trial and error in the choice apparatus. They learn when they make an error.

On a gray background, the trained bees avoided two vertical black lines $1^{\circ}$ wide (Figure 2D), but they avoided the black 
panel even more (Figure 2C), showing that they had learned to avoid the contrast at the vertical edges of the black panel, and also that the black panel was a stronger signal than the two black vertical lines. These results are consistent with the conclusion that the bees detected and learned the strongest signal in the training, at the vertical edges of black on the unrewarded target.

The trained bees strongly preferred a gray panel on white from a gray panel on black (Figure 2F), showing that they had not learned the colors of the panels in the training. They failed to distinguish a gray panel on white from a white panel on gray (Figure $2 \mathrm{G}$ ), showing that they did not detect the white panel, the gray background, or the levels of contrast at the boundaries of the panels. When tested with plain white versus black, they preferred the white, confirming that they avoided strong contrast at vertical edges.

Nothing in these results distinguishes between the actions of the green and the blue receptors or between intensity inputs (tonic) and modulated inputs (phasic). Because the two receptor types respond differently to colors, the trained bees were tested next with equiluminant colored patterns that reduce the number of unknown variables and test the receptor types separately.

\section{White and black on gray were detected as blue plus contrast}

After the same training (Figure 3A), the bees strongly preferred blue on gray to buff on gray, although there was no difference in green content or green contrast (Figure 3B). However, with ultramarine versus hemp on gray, with no blue difference, they failed to distinguish the targets (Figure 3C). They also failed to distinguish gratings with no blue difference (Figure 3D) or no green difference (Figure 3E), showing that they had not measured a difference in blue or green modulation. The first four tests show that to make a full response, the trained bees required the green contrast at the vertical edges of the black training panel as well as the greater content of blue in the white panel.

Confirming this conclusion, when the blue panel was tested versus two black lines that generated green contrast, the trained bees responded very well (Figure 3F). However, when the blue panel and the black bars were on the same target versus plain gray, the preferences for them were almost exactly cancelled (Figure 3G). This test takes us far from the original patterns, but the bees still revealed what they had learned. They had not detected the patterns; they had learned only the responses to the blue and the edges.

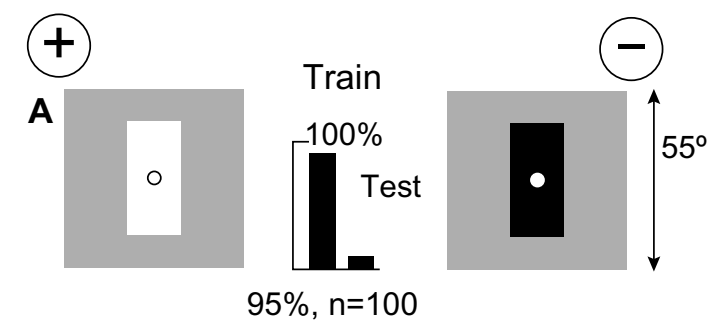

B

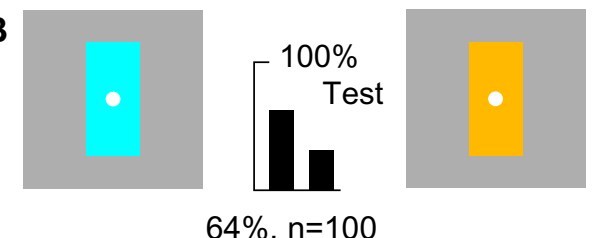

C

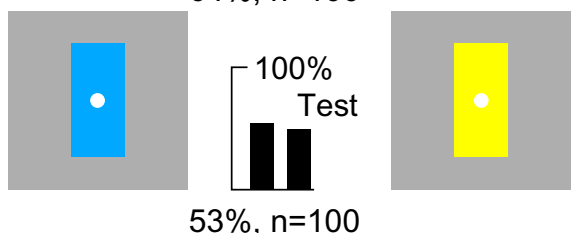

D

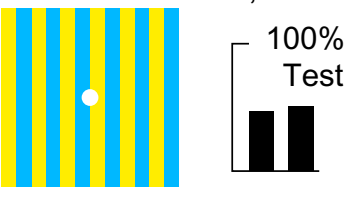

$47 \%, n=100$

E

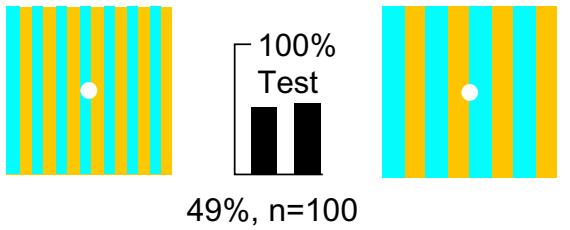

$\mathbf{F}$
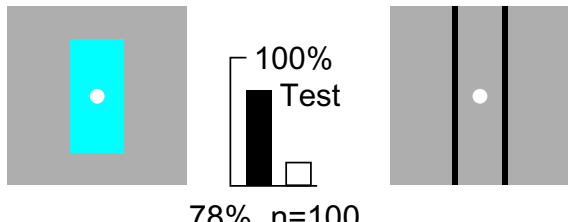

G

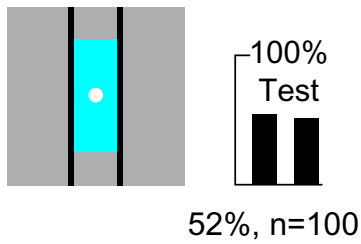

Figure 3 White and black were distinguished by blue content and green modulation. Notes: (A) Training patterns. (B) With no green difference between blue and buff, the bees responded to the blue content. (C) The trained bees failed to distinguish panels that were equiluminant to the blue receptors. (D and $\mathbf{E}$ ) They failed to respond to differences in green or blue modulation. (F) They avoided vertical black lines but went toward the blue area. $(\mathbf{G})$ The black lines canceled the attraction of the blue area.

\section{Bees learn the position of blue in the vertical direction}

The bees were trained on a black panel above a white one versus a white panel above a black one (Figure 4A). When tested with targets that were equiluminant to the green receptors 


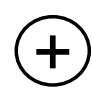

Train

$\mathbf{A}^{\searrow}$
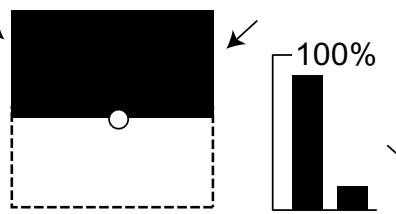

$90 \%, n=200$

B

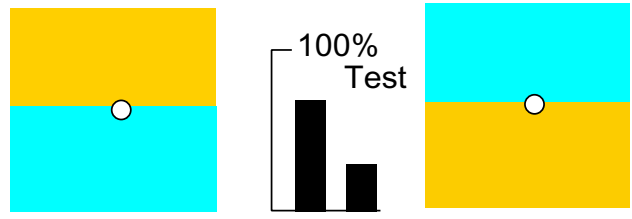

$72 \%, n=100$

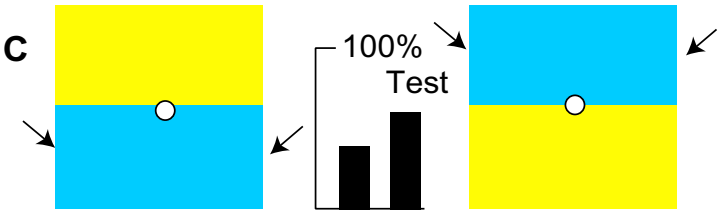

$39 \%, n=100$

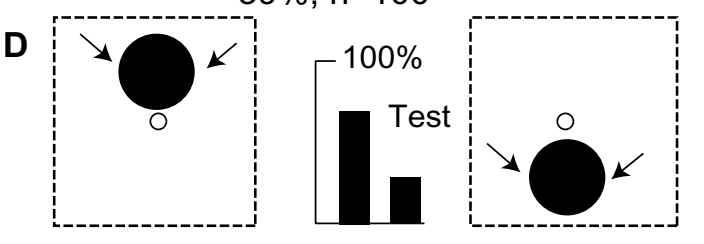

$70 \%, n=100$

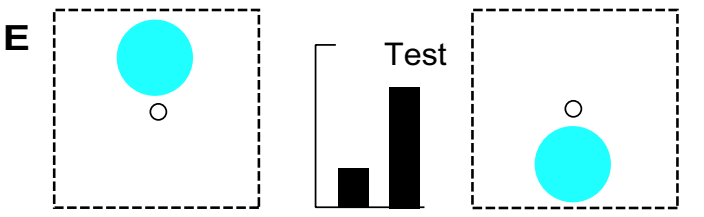

$24 \%, n=100$

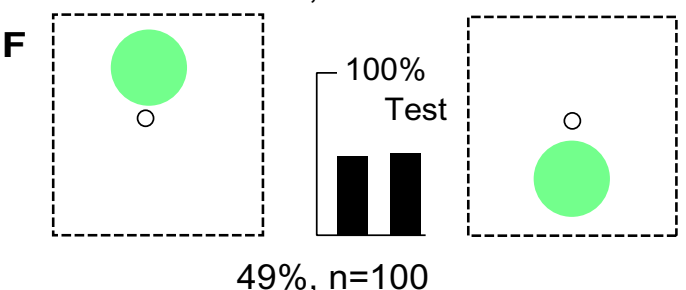

Figure 4 Black panels were distinguished by the positions of green contrast and of blue.

Notes: The small arrows indicate the position of the strongest signal. (A) Training patterns. (B) Blue replaced white in two test patterns that were equiluminant for the green receptors. (C) With no blue contrast, they detected the green contrast at the edges of the ultramarine (arrows). (D) Black spots on white were located by the position of the green contrast (arrows). (E) Preferences were reversed with blue spots on white, showing that background white was only weakly blue. $(\mathbf{F})$ With billiards green spots on white, the positions of the green modulation and blue content cancelled.

(Figure 4B), they responded to the blue content of the white areas in the training, as they did in Figure 3B. They never responded to the green or buff content in the white. With targets of hemp and ultramarine, with no blue difference (Figure 4C), they reversed their performance because they responded to the strong green contrast at the outside edges of the ultramarine. They correctly located the positions of two $20^{\circ}$ black spots on a white background (Figure 4D), by the position of the strong green contrast. Apparently, they ignore the white area because it is continuous with the white interior walls of the apparatus. However, when blue was substituted for black, the strong contrast at the edges of the spots was lost. The bees reversed their preference (Figure 4E). Therefore they had learned the position of the black by the absence of blue, but a small area of blue paper was now a stronger stimulus than a large area of white. The white reflects blue (Table 1), but on a white background in a white apparatus, the response to white had adapted, except where black had been located in the training.

Although the spots were in different positions, the two targets were indistinguishable when the spots were Billiards green on a white background (Figure 4F) because the vertical difference in the position of green contrast cancelled out the vertical difference in the position of the blue content in the green. The trained bees detected the spots, but they could not decide between the conflicting inputs. This looks very strange, but the null was result of the mutual cancellation of two conflicting inputs that had been contrived by the experiment.

\section{With equal white and black, the targets could not be distinguished by color}

The training patterns (Figure 5A) had the same average brightness because each central panel was a half of the area of the whole target. When tested with patterns of buff and blue that were equiluminant to the green receptors and equal in total color (Figure 5B), the trained bees detected no difference, showing that green contrast was essential. With targets of hemp and ultramarine, displaying green contrast but no blue contrast, the trained bees clearly recognized a preferred feature somewhere (Figure 5C). As shown below, this was green contrast at the outer edges. They distinguished the rewarded training pattern from plain gray (Figure 5D), but the unrewarded training target was preferred over plain gray (Figure 5E), showing that they preferred the strongest contrast, at the vertical edges of black. They preferred the more widely separated vertical black edges over those closer together (Figure 5F), confirming that they had measured the angular width to the outer edges of the rewarded training pattern.

\section{The bees distinguish the heights of the spots with blue}

A small group of bees was trained to discriminate between a $20^{\circ}$ white spot at the bottom and a similar spot at the top of a black background (Figure 6A). They were tested with 
$\oplus$

Train

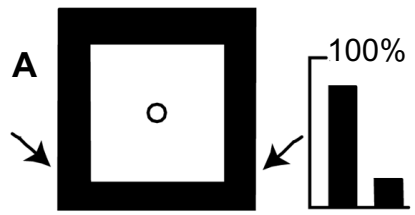

$89.0 \%, \mathrm{n}=200$

B

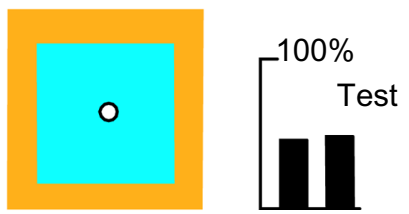

$48.5 \%, n=200$

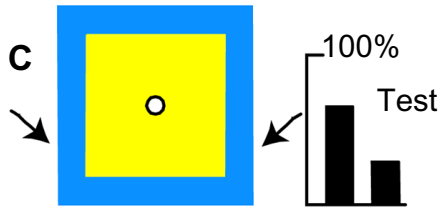

$65.5 \%, n=200$

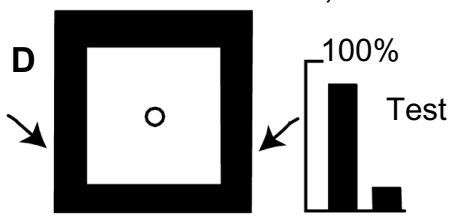

$84.5 \%, n=200$

E

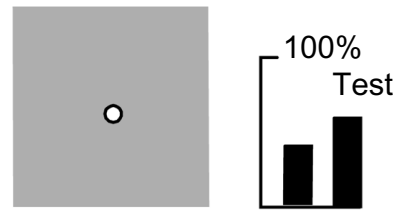

$40 \%, n=100$

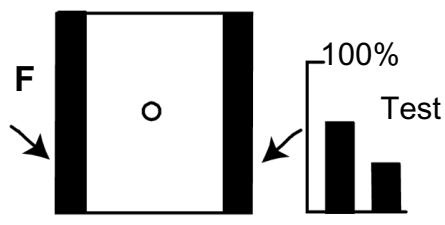

$63 \%, n=100$
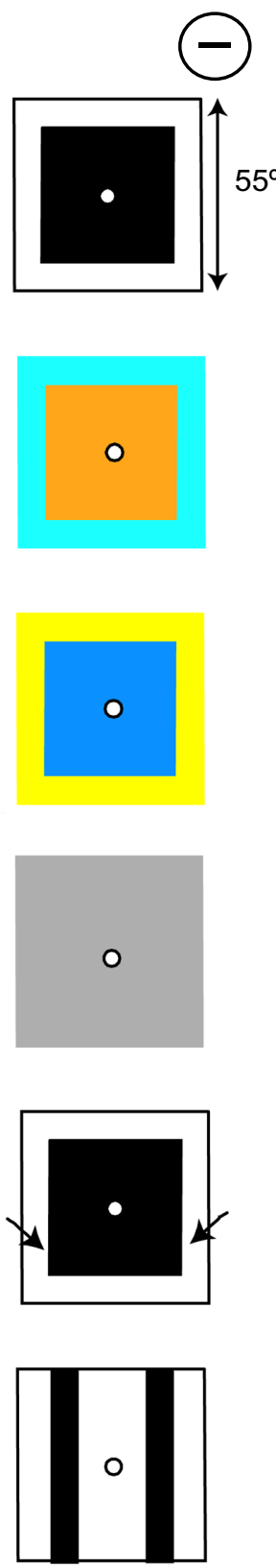

Figure 5 Targets of equal white and black, displayed no color difference.

Notes: (A) Training patterns. (B) The trained bees could not distinguish patterns that were equiluminant to the green receptors. $(\mathbf{C})$ They responded well to green contrast at the edges of patterns that were equiluminant to the blue receptors. (D and E) In the training, they had learned to go to the vertical edge of the wider pattern. (F) They preferred the outer vertical edge to the inner one, as in (A). Arrows show vertical edges.

both training targets versus a plain black, showing that they had learned only the unrewarded one (Figure 6B and C). When tested with blue spots on a black background, they correctly recognized the spot positions (Figure 6D) but failed with hemp spots on an ultramarine background with no blue difference (Figure 6E). When tested with black spots on a white background, they reversed their preference because $\oplus$ Train

A
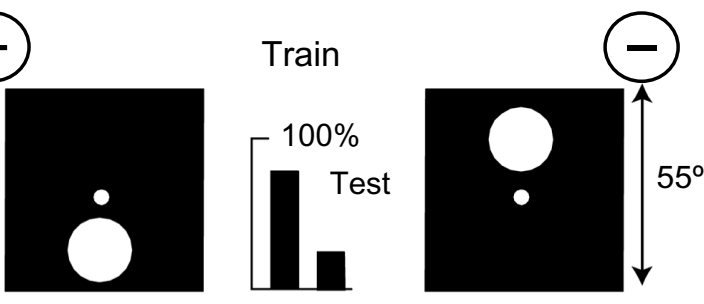

$78 \%, n=100$

B

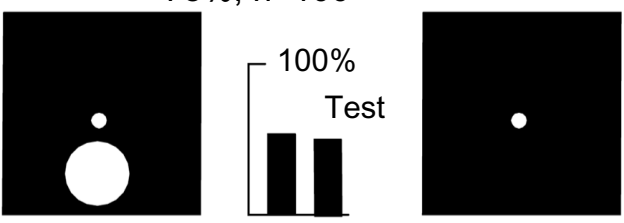

$51 \%, n=100$

C
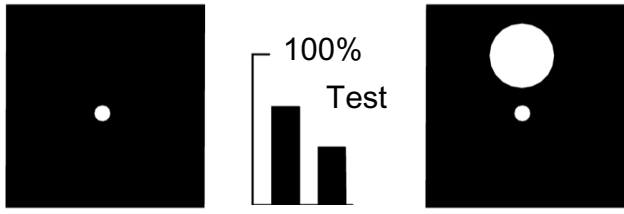

$69 \%, n=100$
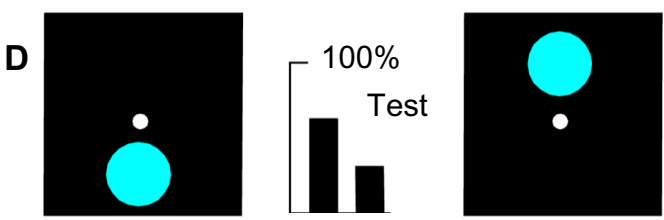

$67 \%, n=100$

E
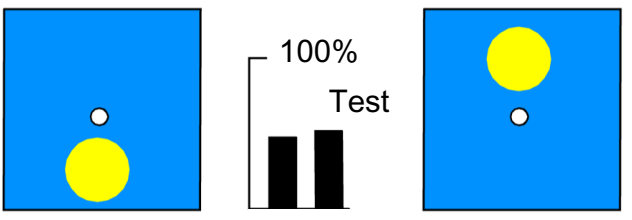

$49 \%, n=100$
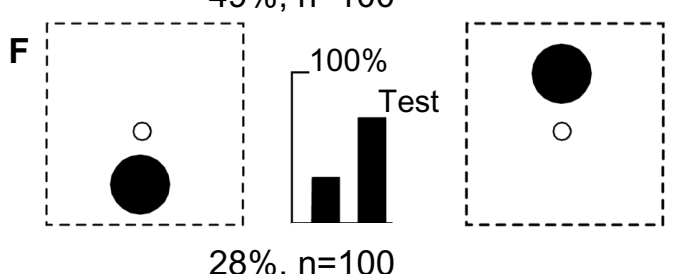

$28 \%, n=100$

Figure 6 Blue was used to detect the position of a white spot on black. Notes: (A) Training patterns. (B and C) The trained bees failed to recognize the rewarded target versus black. (D) Blue spots were distinguished. (E) The trained bees failed to distinguish targets with abundant green contrast but no blue difference. (F) The preference for the positions reversed with black spots on white, which removed blue from its expected retinotopic position.

the average position of blue on the targets was reversed, and that was apparently their only cue.

\section{The bees measure the width}

\section{of large areas of black}

Having now the key to understanding what the bees consistently detected, we can approach the main question. A small 
group of bees were trained to discriminate between plain white and black targets, each subtending $55^{\circ}$ (Figure 7A). They were tested with a white, gray, and black target (in turn) versus a white one with two large vertical bars at the edges (Figure 7B, C, and D). The scores were different for each pair, showing that the progressively smaller difference at the vertical edges had been detected. Bees trained to go to a white target would go to a gray or even a black target (Figure 7C) in preference to one with four black vertical edges. When tested with pairs of bars in different positions (Figure 7E), they avoided contrast at the extreme edges. They had clearly learned the width between black edges separated by $55^{\circ}$.

In a test with hemp versus ultramarine (Figure 7F), with no blue difference, the trained bees avoided the stronger green contrast at the vertical edges of the ultramarine. In a test with buff versus blue, which were equiluminant for the green receptors (Figure 7G), the green modulation was useless, but they preferred the blue that they had learned from the white training target. Once again, the recognition depended on an area displaying blue content and the measure and position of green contrast.

\section{Bees distinguish white from a color by the amount of blue}

To reinvestigate some previous anomalous results,, 314 a small group of bees was trained to discriminate between a green and a plain white target, each subtending $55^{\circ}$ (Figure 8A). The trained bees failed to distinguish the green target from one that looked quite different and displayed no internal green contrast (Figure 8B), showing that the green target had not been recognized. The trained bees also failed to distinguish the green target from a black or hemp one (Figure 8C and D) because these colors lacked the blue content that the bees had learned to avoid, as demonstrated by the excellent discrimination of green and blue (Figure 8E). Whether green modulation was also a cue was not investigated.

\section{Discussion}

Starting with the ability of the bees to distinguish between black and white, we have found that they are not interested in black or white. They avoid the task by detecting the angular width of the black in the horizontal direction, from edge to edge, and the difference in the amount of blue that they detect in the white areas. They also locate black as the absence of blue. In a white apparatus, a panel of white adapts away on a background of white, so that when black is removed, the sudden display of blue has a strong effect (Figure 4D-F and $6 \mathrm{~F})$.
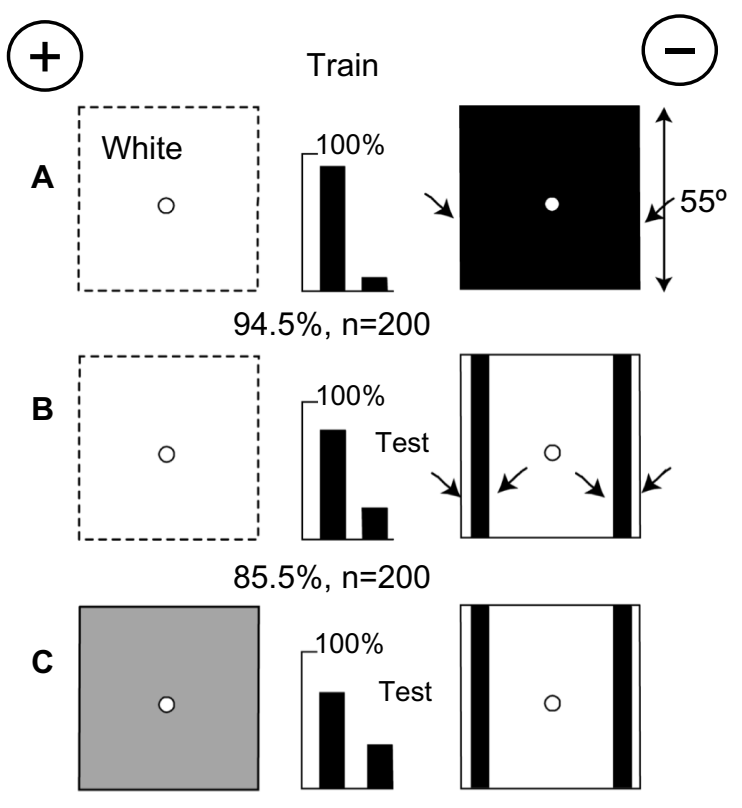

$5 \%, n=200$
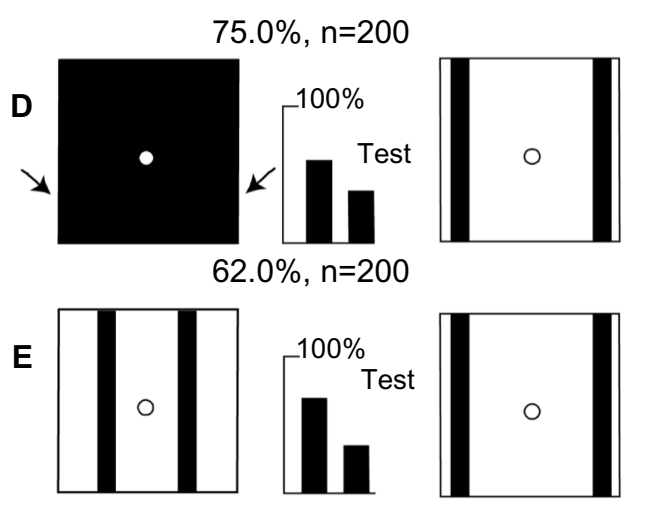

$72.5 \%, n=200$
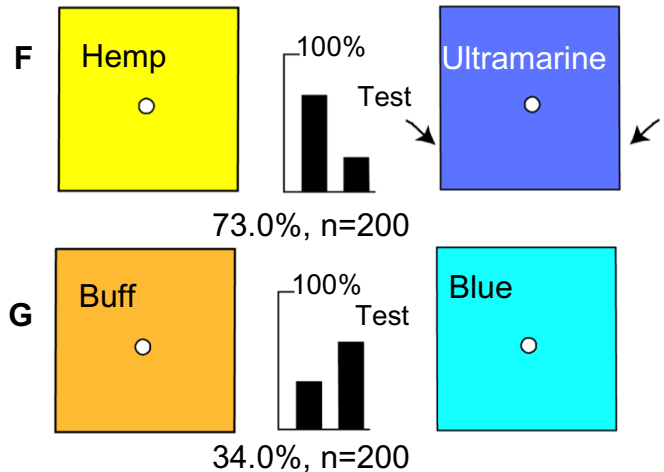

Figure $\mathbf{7}$ The bees measure the width of the green modulation and the position of blue.

Notes: (A) Training patterns. (B-D) Quantitative responses to the differences between green contrast at the edges. (E) They had a memory of the width between the edges in the training. (F) With no blue difference, they avoided the greater green contrast at the edges of the ultramarine. (G) In the absence of green contrast, the trained bees went toward blue, which they learned from the white training target.

Long ago, Hertz ${ }^{12}$ stressed that the most important signal for bees searching for the reward was related to the total amount of edge, which she called "figurale Intesität". Contrast, brightness, and the gray level of areas were of much lower priority. Contrast is a measure of the size and sign of 
$\oplus$

Train, green versus white

A
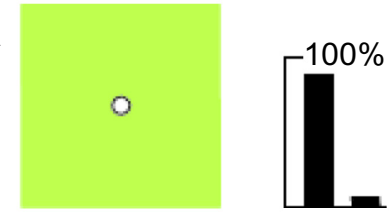

$94.0 \%, n=200$

B

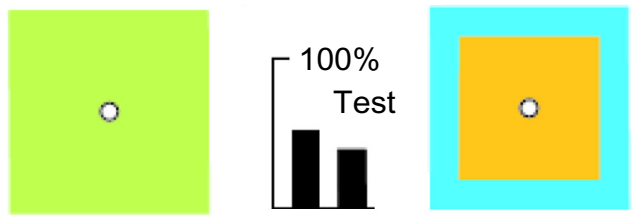

$55.0 \%, n=200$

C
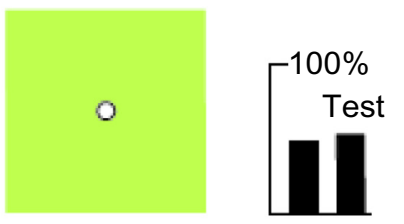

$48.0 \%, n=200$

D
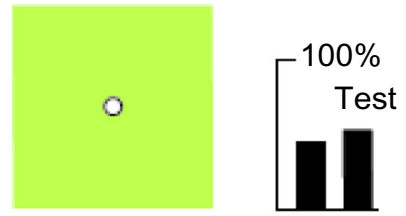

$\circ$

$45.0 \%, n=200$

$\mathbf{E}$

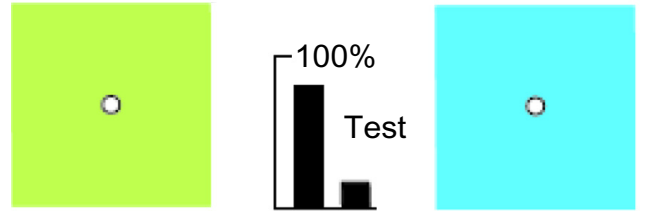

$89.0 \%, n=200$

Figure 8 Bees distinguished green and white by the blue in the white.

Notes: (A) Training targets. (B) A small response avoiding the blue in a pattern with no internal green contrast. (C and D) The green target was not distinguished from plain hemp or black, which displayed little blue. (E) The trained bees avoided the blue learned from the unrewarded target.

the step in the stimulus as a receptor scans across a boundary, relative to the average stimulus. Although it is founded on human vision, the concept is useful for all eyes that move. For bees that scan in the horizontal plane, the signal is the modulation, which can be approximated by contrast $\times$ total length of the vertical edge.

Measures of the signals to each receptor type were calculated from the receptor spectral sensitivity and the distribution of the relative intensity at each wavelength in the emission spectrum of the experimental colored paper (Table 1). These are measures of the stimulus relative to the white paper. For a single receptor type acting monochromatically, this is always stronger, with less noise, than a chromatic signal derived by the subtraction of the response of one receptor type from another. ${ }^{15}$

The other preferred input, the green modulation caused by horizontal scanning of edges or boundaries, was measured as the difference in the receptor excitation derived from vertical edges. The term "modulation" is intended to convey Hertz's idea of figural intensity, which can be approximated by the total length of (vertical) edge multiplied by its contrast. Modulation, in turn, can be related to the responses of the peripheral sensory neurons. The bees simply learned the strongest signal for their phasic detectors, which they find at a vertical edge of a black area, irrespective of which target displayed it. The strongest modulation would be the strongest signal. The behavioral tests confirm that the responses were to figural intensity, not contrast (Figure 2).

The bees also measured the average or total amount of blue in an area, which is in a different dimension from modulation, like the terms in a differential equation. The bees learned the spatial relation between blue and modulation in one or both of the training targets. They never indicated that they had learned a ratio or the contrast between two colors.

A researcher in artificial intelligence would never design a system to distinguish between black and white based on the bee, but the bee is not interested in black or white. There is no reason why bees should use color discrimination as long as they have an effective algorithm for the task at each place. They prefer to use the strongest signal, even though black lines give similar responses as the edges of black areas. To identify the place, the bee learns the strongest modulation signal with small-field green detectors and the angular relation to a blue signal in another location. The coincidence of these two features is learned retinotopically and sometimes has left-right polarity, like a signpost.

In the tests, the trained bees did not somehow compare the test patterns with the memory of one or both training patterns. When they encountered unfamiliar patterns in the tests, they had available only a few features that they had learned, in order of preference, from either one or both training patterns. They responded as best they could and revealed what features they recognized. Their reliance upon only two types of preformed detectors is perfect for a fast discrimination. Repeated as they go, it makes a sparse route map for a brain with a small capacity. Maybe we can learn something from the economical way that the bee codes particular signals from the ever-changing panorama. 


\section{Acknowledgments}

The author thanks Mr Richard Johnston (Canberra Beekeepers Association), for the loan of a hive of bees, and $\mathrm{M}$ Srinivasan, for help with calibrating the reflection spectra of the colored papers in sunlight.

\section{Disclosure}

The author reports no conflict of interest in this work.

\section{References}

1. Autrum H, von Zwehl V. Die spektral Empfindlichkeit einzelner Sehzellen des Bienenauges. Z Vgl Physiol. 1964;48(4):357-384. German.

2. von Frisch K. Der Farbesinn und Formensinn der Biene [Perception of color and form by the bee]. Zool Jahrb Physiol. 1914;35(1):1-188. German.

3. Hertz M. New experiments on colour vision in bees. J Exp Biol. $1939 ; 16(1): 1-8$.

4. Hess C. Beiträge zur Frage nach einen Farbensinne bei Bienen [A contribution to the question of a color sense in bees]. Pflügers Arch. 1918;170(7-9):337-366. German.

5. Laughlin SB, Hardie RC. Common strategies for light adaptation in the peripheral visual systems of fly and dragonfly. J Comp Physiol. 1978;128(5):319-340

6. Srinivasan MV, Laughlin SB, Dubs A. Predictive coding: a fresh view of inhibition in the retina. Proc R Soc Lond B Biol Sci. 1982;216(1205): $427-459$.
7. Horridge A. Pattern vision of the honeybee (Apis mellifera): blue and green receptors in the discrimination of translocation. Neurobiol Learn Mem. 2000;74(1):1-16.

8. Friedlaender M. Zur Bedeutung des Fluglochs im optischen Feld der Biene bei senkrechter Dressuranordnung [The importance of the reward hole in the visual field of bees trained to vertical patterns]. Z Vgl Physiol. 1931;15(2):193-260. German.

9. Horridge A. What Does the Honeybee See? And How Do We Know? Canberra: ANU Press; 2009. Available from: http://epress.anu.edu.au/ honeybee_citation.html. Accessed August 28, 2014.

10. Horridge GA. Visual resolution of gratings by the compound eye of the bee Apis mellifera. J Exp Biol. 2003;206(Pt 13):2105-2110.

11. Horridge GA. Discrimination of single bars by the honeybee (Apis mellifera). Vision Res. 2003;43(11):1257-1271.

12. Hertz M. Über figurale Intensität und Qualitäten in der optische Wahrnehmung der Biene [Figural intensity and quality in the visual perception of the bee]. Biologische Zentralblatte. 1933;53(1):10-40. German.

13. Giger AD, Srinivasan MV. Pattern recognition in honeybees: chromatic properties of orientation analysis. J Comp Physiol A. 1996;178(6): 763-769.

14. Chittka L. Bees, white flowers, and the color hexagon - a reassessment? No, not yet. Naturwissenschaften. 1999;86(8):595-597.

15. Muntz WR. Microelectrode recordings from the diencephalon of the frog (Rana pipiens) and a blue-sensitive system. J Neurophysiol. 1962;25:699-711.
Eye and Brain

\section{Publish your work in this journal}

Eye and Brain is an international, peer-reviewed, open access journal focusing on clinical and experimental research in the field of neuro-ophthalmology. All aspects of patient care are addressed within the journal as well as basic research. Papers covering original research, basic science, clinical and epidemiological studies, reviews and

\section{Dovepress}

evaluations, guidelines, expert opinion and commentary, case reports and extended reports are welcome. The manuscript management system is completely online and includes a very quick and fair peer-review system, which is all easy to use. Visit http://www.dovepress.com/ testimonials.php to read real quotes from published authors. 